\title{
Effect of surface modification on single-walled carbon nanotube retention and transport in saturated and unsaturated porous media
}

Yuan Tian, Bin Gao, Verónica L. Morales, Yu Wang1, and Lei Wu

NOTICE: this is the author's version of a work that was accepted for publication in Journal of Hazardous Materials. Changes resulting from the publishing process, such as peer review, editing, corrections, structural formatting, and other quality control mechanisms may not be reflected in this document. Changes may have been made to this work since it was submitted for publication. A definitive version was subsequently published in Journal of Hazardous Materials, [Vol. 239-240, (2012)] DOI http://dx.doi.org/10.1016/j.jhazmat.2012.09.003 
Effect of surface modification on single-walled carbon nanotube retention and transport in saturated and unsaturated porous media

\author{
Yuan Tian ${ }^{1}$, Bin Gao ${ }^{1 *}$,Verónica L. Morales ${ }^{2}$, Yu Wang ${ }^{1}$, and Lei Wu ${ }^{1}$
}

1. Department of Agricultural and Biological Engineering, University of Florida, Gainesville, FL 32611-0570

2. SIMBIOS Centre, University of Abertay, Dundee, DDI U.K.

*Corresponding Author, Phone: (352) 392-1864 ext.285, email: bg55@ufl.edu 


\begin{abstract}
This work investigated the effect of different surface modification methods, including oxidization, surfactant coating, and humic acid coating, on single-walled carbon nanotube (SWNT) stability and their mobility in granular porous media under various conditions. Characterization and stability studies demonstrated that the three surface modification methods were all effective in solubilizing and stabilizing the SWNTs in aqueous solutions. Packed sand column experiments showed that although the three surface medication methods showed different effect on the retention and transport of SWNTs in the columns, all the modified SWNTs were highly mobile. Compared with the other two surface modification methods, the humic acid coating method introduced the highest mobility to the SWNTs. While reductions in moisture content in the porous media could promote the retention of the surface modified SWNTs in some sand columns, results from bubble column experiment suggested that only oxidized SWNTs were retention in unsaturated porous media through attachment on air-water interfaces. Other mechanisms such as grain surface attachment and thin-water film straining could also be responsible for the retention of the SWNTs in unsaturated porous media. An advectiondispersion model was successfully applied to simulate the experimental data of surface modified SWNT retention and transport in porous media.

Keywords: carbon nanotube, solubilization, environmental fate, mobility, model
\end{abstract}




\section{Introduction}

Because of their exceptional electrical, mechanical and thermal properties, carbon nanotubes (CNTs) have been widely manufactured and used in many applications [1]. This may result in unintended and/or accidental releases of CNTs into the natural environment. Upon release, CNTs could be transported by water flow through the vadose zone and cross the water table to reach the groundwater and drinking water aquifers. Several studies have found that bioaccumulation and bioaugumentation of CNTs by the food chain may impose great risks to the ecosystems and the public health $[2,3]$. Because CNTs have a strong affiliation to many contaminants, such as heavy metals and organic compounds, their potential risks in soil and groundwater systems could be aggravated [4, 5]. Therefore, fate and transport of CNTs in porous media need thorough investigations.

Pristine CNTs have large surface areas and strong van der Waals attraction forces among individual particles, inevitably causing their unwanted self-aggregations in aqueous solutions. Various surface modification methods thus have been developed to enhance CNTs dispersion in solutions to facilitate their applications. The most common CNT surface modification methods include either strong oxidative treatments or use of amphiphilic polymers or surfactants. Surface oxidization with strong acids can introduce hydrophilic functional groups (e.g. carboxyl and hydroxyl groups) to the CNTs surfaces to help their dispersion [6]. Amphiphilic polymers or surfactants, such as natural organic matter (NOM) treatment and ionic surfactants, can wrap around and solubilize CNTs in aqueous solutions [7]. The surface modification methods in general can introduce surface charges to the CNTs and thus electrostatic repulsion forces to reduce their aggregation in aqueous solutions. As a result, dispersed or solubilized CNTs may have a higher mobility 
in soil and groundwater systems than the pristine CNTs. Although several studies have been conducted to examine the fate and transport of various types of CNTs in porous media [8-14], little research effect has been made to determine the effect of different surface modifications on CNT retention and transport in porous media, particularly under unsaturated conditions.

In this work, laboratory experiments were performed to investigate the retention and transport of surface modified single-walled carbon nanotubes (SWNTs) in porous media. Three types of SWNTs with different surface modifications were tested in this work: acid oxidized, humic acid (HA) dispersed, and sodium dodecylbenzene sulfonate (SDBS, anionic surfactant) dispersed. Stabilities of the three types of SWNTs in aqueous solutions were compared. Columns packed with quartz sand with different combinations of grain sizes and moisture contents were used to compare the breakthrough behaviors of the surface modified SWNTs. In addition, bubble column experiments were conducted to determine the interactions between the modified SWNTs and air-water interfaces.

The overarching objective of this work was to determine the effect of different surface modification methods on the retention and transport of SWNTs in saturated and unsaturated porous media. Specific objectives were to: 1) evaluate effectiveness of the three surface modification methods in stabilizing SWNTs in aqueous solutions; 2) determine the interactions between the three types of SWNTs and the air-water interfaces; 3) compare the retention and transport of the three types of SWNTs in saturated porous media of different grain sizes; and 4) compare the retention and transport of the three types of dispersed SWNTs in unsaturated porous media of different combinations of moistures and grain sizes. 


\section{Materials and methods}

\subsection{Surface modified SWNTs}

Pristine SWNTs were obtained from Cheap Tubes Inc. (Brattleboro, VT) with diameters of $1.0-2.0 \mathrm{~nm}$ and lengths of $500-2,000 \mathrm{~nm}$ as reported by the manufacturer. The pristine SWNTs were used in this work to make all the three surface modified SWNTs.

Surface oxidized SWNTs (O-SWNTs) were prepared from the pristine SWNTs using a 3 to 1 ratio of sulfuric and nitric acids to add hydrophilic surface functional groups (e.g., hydroxyl and carboxyl). To prepare the suspensions for experimentation, 16 mg O-SWNTs were suspended in $1000 \mathrm{ml}$ deionized (DI) water, followed by $30 \mathrm{~min}$ of ultrasonication (Misonix S3000).

Surfactant and HA dispersed SWNTs (S-SWNTs and H-SWNTS) were prepared using of SDBS and HA solutions, respectively. SDBS (25155-30-0) and HA (Elliott Soil HA Standard, 1S102H) were obtained from Sigma-Aldrich Co. (St. Louis, MO) and International Humic Substances Society (St. Paul, MN, USA), respectively. The SWNT suspensions were prepared by mixing $20 \mathrm{mg}$ of pristine SWNTs with $200 \mathrm{~mL}$ of the SDBS solution ( $1 \%$ by weight) or the HA solution $(5 \mathrm{mg} / \mathrm{L})$, followed the method reported by O'Connell et al. [15]. High-shear homogenization (IKA T-25 Ultra-Turrax) for $1.5-2.0 \mathrm{~h}$ and ultrasonication (Misonix S3000) for $10 \mathrm{~min}$ were used to aid dispersion. The resulted SWNT suspensions were then diluted to a concentration of 16 $\mathrm{mg} / \mathrm{L}$ for future uses. 
Electrophoretic mobility (EPM) of the surface modified MWNTs was determined using a ZetaPlus (Brookhaven Instrument Co., Holtsville, NY) and the Smoluchowski's model was used to estimate their zeta potential [8]. Fourier transform infra-red (FTIR) analysis was conducted to characterize the surface functional groups of the pristine and oxidized SWNTs. To obtain observable adsorption spectra, each of the SWNTs was mixed with potassium bromide powder at a weight ratio of $0.1 \%$ and then the mixtures were pressed into pellets. The spectra were measured using a Bruker Vector 22 FTIR spectrometer (OPUS 2.0 software).

\subsection{Porous media}

Quartz sand (Standard Sand \& Silica Co.) was used in this study and was sieved into three different size distributions: fine $(0.1-0.2 \mathrm{~mm})$, medium $(0.5-0.6 \mathrm{~mm})$, and coarse $(1.4-1.6 \mathrm{~mm})$. The sand was then washed sequentially by tap water, $10 \%$ nitric acid (v:v) and deionized water, and then baked at $550{ }^{\circ} \mathrm{C}$ to remove the metal oxides and organic impurities. The zeta potential of the acid-cleaned quartz sand was determined according to the procedure as reported by Johnson et al. [16].

\subsection{Bubble column experiment}

Bubble column experiment was used to examine the interactions between the SWNT particles and the air-water interface. In brief, an acrylic column measuring $2.5 \mathrm{~cm}$ in diameter and $100 \mathrm{~cm}$ in height was filled with each of the different surface modified SWNT suspensions. Air was pumped into the chamber at the bottom of the column, resulting in relatively uniform air bubbles rising from the bottom to the top. SWNT 
samples were obtained at different time intervals from the eight sampling ports positioned at different depths to determine the temporal and spatial distribution of SWNTs in the bubble column. Details of the design and procedure of the bubble column experiment were reported previously [11].

\subsection{Sand column experiment}

Column studies were performed to investigate the retention and transport of the surface modified SWNT suspensions in porous media with different grain size and moisture content combinations (Table 2). The quartz sand (i.e., fine, medium, or coarse) was wet-packed (i.e., saturated) into an acrylic column measuring $2.5 \mathrm{~cm}$ in diameter and $15 \mathrm{~cm}$ in height. Six vent holes were drilled on opposite sides at 3, 7.5, and $12 \mathrm{~cm}$ from the top of the column. The vent holes were sealed with gas-permeable porous PTFE membranes (Milliseal Disk, Millipore) to allow air to enter under unsaturated conditions. The moisture content of the unsaturated column was then generated by the gravimetric method. Two-level unsaturated conditions were generated for fine, medium, and coarse sand, respectively. Moisture contents in the saturated and unsaturated columns were shown in the Table 2. Detailed information about column preparations can be found in previous study [11].

Once the column moisture stabilized, background solution of the tested SWNT suspension was first pumped through the column for about three pore volumes (PVs) to stabilize the bulk fluid chemistry. The breakthrough experiment was then initiated by switching from background solution to the SWNT suspension. The SWNT suspension was applied to the column for more than three PVs. The Effluent samples were collected 
from the bottom of the column using a fraction collector (IS-95 Interval Sampler, Spectrum Chromatography, Houston, TX) during the breakthrough experiment to analyze SWNT concentrations with a UV-Vis Spectrophotometer (Evolution 60, Thermo Scientific) at a wavelength of $245 \mathrm{~nm}$ [11].

Only one pump was used at the outflow end of the column to regulate the flow rate in experiments with the water-saturated column. The influent concentrations of SWNTs used in the saturated experiments matched those of the unsaturated experiments. The procedures of sample collection and analysis for saturated experiments were the same as those for the unsaturated experiments.

\subsection{Mathematical model}

The advection-dispersion equation (ADE) coupled with kinetic expressions was used to simulate the retention and transport of the SWNTs in the sand columns. The governing equation can be written as [17]:

$$
\frac{\partial C}{\partial t}=D \frac{\partial^{2} C}{\partial z^{2}}-v \frac{\partial C}{\partial z}-k C
$$

where $C$ is the concentration of suspended SWNTs in pore water $\left(\mathrm{M} \mathrm{L}^{-3}\right), t$ is time $(\mathrm{T}), z$ is the travel distance in the direction of flow $(\mathrm{L}), D$ is the dispersion coefficient $\left(\mathrm{L}^{2} \mathrm{~T}^{-1}\right), v$ is the average linear pore-water velocity $\left(\mathrm{L} \mathrm{T}^{-1}\right)$, and $k$ is the deposition rate $\left(\mathrm{T}^{-1}\right)$. This equation can be solved analytically with a zero initial concentrations, a pulse-input boundary condition, and a zero-concentration-gradient condition at the outlet boundary $[18,19]$. 


\section{Results and discussion}

\subsection{Characteristics and stability of surface modified SWNTs}

The FTIR spectra were used to determine the acid oxidation effect on the O-SWNTs. Compared to the pristine SWNTs, the O-SWNTs showed more FTIR peaks in the spectra (Figure 1). As shown in the Figure, peaks at 3445, 1635, $1045 \mathrm{~cm}^{-1}$ of the O-SWNTs could be assigned to the $\mathrm{O}-\mathrm{H}$ stretch from carboxyl groups $(\mathrm{O}=\mathrm{C}-\mathrm{OH}$ and $\mathrm{C}-\mathrm{OH})$, the $\mathrm{C}=\mathrm{O}$ bond, and the $\mathrm{C}-\mathrm{O}$ bond, respectively [20]. This indicates that the acid oxidation processes introduced carboxyl and hydroxyl functional groups to SWNT surfaces.

The EPM and zeta-potential values of the tested SWNTs were all negative (Table 1), confirming that their surfaces were coated with the negatively charged functional groups, SDBS, or HA under the tested experimental conditions. The absolute values of the EPM and zeta-potential followed the order of H-SWNTs > S-SWNTs > O-SWNTs, suggesting the same trend for their stability. The structure and composition of HA were comprised with numerous functional groups, which tend to bring more negative charges to the surface of H-SWNTs, enhancing the repulsive electric double layer forces to separate SWNT bundles into individual ones, and thus facilitating the dispersion of H-SWNTs in aqueous solutions. Similarly, functional groups and surfactants on the surfaces of the modified SWNTs also introduces negative charges, but less than that of the H-SWNTs, to help the dispersion/solubilization.

Temporal changes of the surface modified SWNT concentrations are shown in Figure 2. The relative concentrations of the S-SWNTs and H-SWNTs remained unchanged for $24 \mathrm{~h}$, indicating strong stability due to the surface modification. The concentrations of the O-SWNTs was unchanged for the first $10 \mathrm{~h}$, but decreased slightly 
(3\%) from 10 to $24 \mathrm{~h}$. This suggests that although all the surface modification methods were effective, the HA and SDBS modifications might have slightly better effect than the acid oxidization method to disperse and stabilize SWNTs in solutions. To avoid the potential interferences from aggregations of the surface modified SWNTs, all transport experiments used fresh prepared SWNTs suspension. In addition, each experiment, including sample collections and analyses, was controlled to be completed within $10 \mathrm{~h}$.

\subsection{Interaction between surface modified SWNTs and air bubbles}

The temporal changes of concentrations of the surface modified SWNTs at the eight vertically distributed sampling ports (P1-8 from top to bottom) are shown in Figure 3. All the reported concentrations were normalized to the initial concentration. Concentrations of S-SWNT and H-SWNT from the eight sampling ports within the bubble column stayed unchanged for more than $20 \mathrm{~h}$ with continuous air bubble injections. This result suggests that the surfactant or the humic acid dispersed SWNTs do not attach to the air-water interface under the tested experimental conditions, which is consistent with the results reported previously [11]. The O-SWNTs, however, showed a different concentration distribution in the bubble column after $20 \mathrm{~h}$. At the end of the experiment, the O-SWNT concentration at P1 (top) increased about 5\%, but decreased about 10\% at P8. This result indicates that O-SWNTs were attached to the air bubbles and transferred from the bottom of the bubble column to the top.

\subsection{Transport of surface modified SWNTs in medium sand}

Breakthrough curves of the three types of surface modified SWNTs in saturated and 
unsaturated columns packed with medium sized sands are shown in the Figure 4. The breakthrough points of all the modified SWNTs were less than $1 \mathrm{PV}$, reflecting the particle dispersion effect and possible enhanced particle velocity caused by the size exclusion effect [21]. Under saturated conditions, the three types of SWNTs showed similar transport behaviors in the sand columns. After applied to the columns, the relative concentrations (i.e., $\mathrm{C} / \mathrm{C}_{0}$ ) of the three modified SWNTs quickly climbed to a peak at one, indicating all three surface modification methods are effective in enhancing the mobility of SWNTs in porous media. For S-SWNTs and H-SWNTs, the moisture contents did not show notable influence on their breakthrough behaviors and they had almost the same breakthrough curves for all the tested conditions (Figure 4). For O-SWNTs, however, the peak breakthrough concentrations under moisture content of 0.21 and 0.13 were about $4 \%$ and $12 \%$ lower than that of saturated (0.39) experiment, respectively. This indicates that the O-SWNTs were less mobile than the H-SWNTs and S-SWNTs in unsaturated medium porous media. Those results are consistent with the results obtained from the bubble column experiment that the air-water interface (AWI) can capture (retain) the OSWNTs but not the S-SWNTs or the H-SWNTs.

Previous studies have demonstrated that colloidal and nanosized particles could be retained in unsaturated porous media through irreversibly deposited on the AWI under certain conditions [22, 23]. Although electrostatic forces between negatively charged particles and the AWI are repulsive and unfavorable for attachment [24, 25], hydrophobic and capillary forces could dominate the interactions between the particles and the AWI $[24,26,27]$. 


\subsection{Transport of surface modified SWNTs in fine sand}

Breakthrough curves of the surface modified SWNTs in the fine sand columns under different moisture conditions are shown in Figure 5. The peak breakthrough concentrations of H-SWNTs in all find sand columns were close to one under the three moisture conditions, indicating no/little H-SWNTs was retained in the porous media. Small portions of retention for O-SWNTs and S-SWNTs were observed in the fine sand under various moisture conditions (Figure 5). Similar to the results in the medium sand columns, the O-SWNTs had the lowest breakthrough (i.e., lowest mobility) in the fine sand. The H-SWNTs again were highly mobile and had a slightly higher mobility than the S-SWNTs in the fine sand.

Under statured conditions, the retentions of O-SWNTs and S-SWNTs in the fine sand columns could be attributed to their attachment on the grain surfaces. The attachment of CNTs on sand grain surfaces is mainly controlled by the DLVO or the extended DLVO forces, which are affected by the solution chemistry and physiochemical characteristics of the particle and grain surfaces [28, 29]. Although the experimental conditions were chemically unfavorable, the fine sand had less negatively charged surfaces than the medium or the coarse sand (Table 1). In addition, the surface charge (negative) of the O-SWNTs and S-SWNTs was lower than that of the H-SWNTs (Table 1). As a result, it might be easier for the O-SWNTs and the S-SWNTs to attach to the fine sand surfaces through either second minimum deposition or primary minimum deposition after overriding the energy barrier [13].

Reductions in moisture content showed little impact on the transport of H-SWNTs and S-SWNTs in the fine sand columns (Figure 5), which is consistent with the results 
from the bubble column experiments. While there was no retention of H-SWNTs in the two unsaturated columns, the peak breakthrough concentrations of S-SWNTs in the two unsaturated fine sand column were around $0.92 \mathrm{C}_{0}$, similar to that in the saturated one. This suggests that moisture had no impact on S-SWNT retention and transport; therefore, the retention of the S-SWNTs in the unsaturated fine sand columns can also be attributed to the attachment on the grain surfaces. The transport of the O-SWNTs in the unsaturated fine sand, particularly in the one with moisture of 0.29 , showed lower breakthrough than that in the saturated fine sand (Figure 5), suggesting O-SWNTs may be captured by both grain surfaces and the air-water interfaces. Compared to the unsaturated medium sand, the moisture contents of the unsaturated fine sand were higher. As a result, the air-water interfaces showed lower impact on the transport and retention of O-SWNTs in the fine sand than that in the medium sand.

\subsection{Transport of surface modified SWNTs in coarse sand}

The transport of the three surface modified SWNTs in saturated coarse sand was similar to each other with peak concentrations close to one (Figure 6), indicating no/little deposition on the grain surfaces. This is consistent with the zeta-potential measurements that the coarse sand had the highest negative surface charges.

The two moisture contents used in the unsaturated coarse sand columns (i.e., 0.11 and 0.09) were lower than those in the unsaturated medium (i.e., 0.21 and 0.13 ) and fine (i.e., 0.33 and 0.29) sand columns (Table 2). The relatively low moisture contents in the unsaturated coarse sand reduced the mobility of all the surface modified SWNTs (Figure 6). Compared to the other two SWNTs, the H-SWNTs still had the highest mobility, but 
their breakthrough under low moisture conditions were lower than that under saturated conditions (Figure 6), which were not observed in fine or medium sand columns (Figures 4 and 5). Similarly, low moisture content also lowered the breakthrough of S-SWNTs and O-SWNTs in the unsaturated coarse sand columns.

The retention of S-SWNTs and H-SWNTs in the unsaturated coarse sand columns could be attributed to the water-film straining mechanism [11]. The water-film straining mechanism is controlled by multiple factors and must satisfy two conditions: 1) moisture content is lower than a "critical saturation", at which the connections between pendular rings in the porous media are broken; and 2) water-film thickness is smaller than the particle sizes [30]. For nano-sized particles, such as CNTs, water-film straining only occurs at low moisture conditions [11], and thus no film straining was observed for the three SWNTs in the medium and the fine sand columns because their moisture was relatively high. The transport of the O-SWNTs in the unsaturated coarse sand was lower than that of the other two SWNTs, probably due to combined retention effects from both film training and air-water interface attachment mechanisms.

\subsection{Model simulations}

The ADE model was used to simulate the transport behavior of SWNTs in the sand packed column with various moisture contents and the simulations matched the experimental breakthrough curves very well with $R^{2}$ bigger than 0.99 (Figure 4-6). Peclet number $\left(P e=\frac{v H}{D}\right.$, where $H$ is the column length), Damkohler number $\left(D a=\frac{k H}{v}\right)$ and maximum travel distance ( $L_{\max }=-\frac{v}{k} \ln \frac{C}{C_{0}}$, where $C / C_{0}$ is 0.001 ) were then 
calculated (Table 2). The $P e$ values were relatively large (10-101) indicating flow advection may play more important role in mass transfer of the surface modified SWNTs in the sand columns than dispersion [31]. The $D a$ values were relatively small (0.0010.250), indicating the time-scale for deposition mass transfer was larger than that for advective transport. Compared to the O-SWNTs and the S-SWNTs, the $D a$ values of the H-SWNTs were generally smaller under the same conditions, confirming that the experimental results that H-SWNTs were more mobile than the other two. In addition, the values of $L_{\max }$ for H-SWNTs were larger than those for C-SWNTs and S-SWNTs for most of the tested conditions, further confirming that H-SWNTs had the highest mobility in the porous media.

\section{Conclusions}

In this work, laboratory experiments were conducted to investigate the retention and transport of three types of surface modified SWNTs (i.e., surface oxidization, surfactant modified, and HA modified) in saturated and unsaturated columns packed with fine, medium, or coarse sand. We found that all the three surface modification methods were effective in solubilizing the SWNTs. The absolute values of the EPM and zeta-potential followed the order of H-SWNTs > S-SWNTs > O-SWNTs, which is consistent with their stability trend. While all three surface modified SWNTs were highly mobile in the packed sand columns with different combinations of grain sizes and moisture, the $\mathrm{H}$ SWNTs showed the highest mobility for most of the tested conditions. NOMs, such as humic acid, are ubiquitous in soil and groundwater environment, as well as other aquatic systems. When CNTs are released in the environment, therefore, they may have high 
mobility in water flow due to the presence of NOMs.

The results from this work also suggest that the retention and transport of SWNTs in porous media are controlled by both dispersion/solubilization methods and environmental conditions. Single or combined retention mechanisms, including gain surface attachment, air-water interface attachment, and thin-water film straining, may be responsible for the retention of the surface modified SWNTs in the saturated and unsaturated porous media depending on the experimental conditions. In addition, we also found that the advectiondispersion model could be used to simulate the retention and transport of surface modified SWNTs in porous media for both saturated and unsaturated conditions.

\section{Acknowledgements}

This work was partially supported by the NSF through grant CBET-1054405. 


\section{References}

[1] M.S. Mauter, M. Elimelech, Environmental Applications of Carbon-Based Nanomaterials, Environ. Sci. Technol. 42 (2008) 5843-5859.

[2] N.A. Monteiro-Riviere, R.J. Nemanich, A.O. Inman, Y.Y.Y. Wang, J.E. Riviere, Multi-walled carbon nanotube interactions with human epidermal keratinocytes, Toxicol. Lett. 155 (2005) 377-384.

[3] M.A. Chappell, A.J. George, K.M. Dontsova, B.E. Porter, C.L. Price, P. Zhou, E. Morikawa, A.J. Kennedy, J.A. Steevens, Surfactive stabilization of multi-walled carbon nanotube dispersions with dissolved humic substances, Environ. Pollut. 157 (2009) 1081-1087.

[4] G.P. Rao, C. Lu, F. Su, Sorption of divalent metal ions from aqueous solution by carbon nanotubes: A review, Sep. Purif. Technol. 58 (2007) 224-231.

[5] S.A. Kumar, S.F. Wang, Adsorption of ciprofloxacin and its role for stabilizing multiwalled carbon nanotubes and characterization, Mater. Lett. 63 (2009) 1830-1833.

[6] B. Smith, K. Wepasnick, K.E. Schrote, H.-H. Cho, W.P. Ball, D.H. Fairbrother, Influence of Surface Oxides on the Colloidal Stability of Multi-Walled Carbon Nanotubes: A Structure-Property Relationship, Langmuir. 25 (2009) 9767-9776.

[7] M. Li, C.P. Huang, Stability of oxidized single-walled carbon nanotubes in the presence of simple electrolytes and humic acid, Carbon 48 (2010) 4527-4534.

[8] P. Wang, Q.H. Shi, H.J. Liang, D.W. Steuerman, G.D. Stucky, A.A. Keller, Enhanced Environmental Mobility of Carbon Nanotubes in the Presence of Humic Acid and Their Removal from Aqueous Solution, Small 4 (2008) 2166-2170. 
[9] X.Y. Liu, D.M. O'Carroll, E.J. Petersen, Q.G. Huang, C.L. Anderson, Mobility of Multiwalled Carbon Nanotubes in Porous Media, Environ. Sci. Technol. 43 (2009) 8153-8158.

[10] D. Bouchard, W. Zhang, T. Powell, U.S. Rattanaudompol, Aggregation Kinetics and Transport of Single-Walled Carbon Nanotubes at Low Surfactant Concentrations, Environ. Sci. Technol. 46 (2012) 4458-4465.

[11] Y.A. Tian, B. Gao, K.J. Ziegler, High mobility of SDBS-dispersed single-walled carbon nanotubes in saturated and unsaturated porous media, J. Hazard. Mater. 186 (2011) $1766-1772$.

[12] Y.A. Tian, B. Gao, C. Silvera-Batista, K.J. Ziegler, Transport of engineered nanoparticles in saturated porous media, J. Nanopart. Res. 12 (2010) 2371-2380.

[13] Y. Tian, B. Gao, L. Wu, R.M. Carpena, Q. Huang, Effect of solution chemistry on multi-walled carbon nanotube deposition and mobilization in clean porous media, J. Hazard. Mater. (2012) doi: 10.1016/j.jhazmat.2012.06.039.

[14] Y. Tian, B. Gao, Y. Wang, V.L. Morales, R.M. Carpena, Q.G. Huang, L.Y. Yang, Deposition and transport of functionalized carbon nanotubes in water-saturated sand columns, J. Hazard. Mater. 213 (2012) 265-272.

[15] M.J. O'Connell, S.M. Bachilo, C.B. Huffman, V.C. Moore, M.S. Strano, E.H. Haroz, K.L. Rialon, P.J. Boul, W.H. Noon, C. Kittrell, J.P. Ma, R.H. Hauge, R.B. Weisman, R.E. Smalley, Band gap fluorescence from individual single-walled carbon nanotubes, Science 297 (2002) 593-596. 
[16] P.R. Johnson, N. Sun, M. Elimelech, Colloid transport in geochemically heterogeneous porous media: Modeling and measurements, Environ. Sci. Technol. 30 (1996) 3284-3293.

[17] J. Smith, B. Gao, H. Funabashi, T.N. Tran, D. Luo, B.A. Ahner, T.S. Steenhuis, A.G. Hay, M.T. Walter, Pore-scale quantification of colloid transport in saturated porous media, Environ. Sci. Technol. 42 (2008) 517-523.

[18] M.T. van Genuchten, W.J. Alves, Analytical solutions of the one-dimensional convective-dispersive solute transport equation, Technical Bulletion No. 1661, U.S. Department of Agriculture. (1982) p 151.

[19] N. Toride, F.J. Leij, M.T. Van Genuchten, The CXTFIT code for estimating transport parameters from laboratory or field tracer experiments, Research Report No. 137, USDA Salinity Laboratory, Riverside, CA. (1995) p 121.

[20] L. Wang, S. Feng, J. Zhao, J. Zheng, Z. Wang, L. Li, Z. Zhu, A facile method to modify carbon nanotubes with nitro/amino groups, Appl. Surf. Sci. 256 (2010) 60606064.

[21] D. Grolimund, M. Elimelech, M. Borkovec, K. Barmettler, R. Kretzschmar, H. Sticher, Transport of in situ mobilized colloidal particles in packed soil columns, Environ. Sci. Technol. 32 (1998) 3562-3569.

[22] B. Gao, J.E. Saiers, J.N. Ryan, Deposition and mobilization of clay colloids in unsaturated porous media, Water Resour. Res. 40 (2004) W08602.

[23] B. Gao, J.E. Saiers, J. Ryan, Pore-scale mechanisms of colloid deposition and mobilization during steady and transient flow through unsaturated granular media, Water Resour. Res. 42 (2006) W01410. 
[24] B. Gao, T.S. Steenhuis, Y. Zevi, V.L. Morales, J.L. Nieber, B.K. Richards, J.F. McCarthy, J.Y. Parlange, Capillary retention of colloids in unsaturated porous media, Water Resour. Res. 44 (2008) W04504.

[25] J.T. Crist, Y. Zevi, J.F. McCarthy, J.A. Throop, T.S. Steenhuis, Transport and retention mechanisms of colloids in partially saturated porous media, Vadose Zone J. 4 (2005) 184-195.

[26] N. Chatterjee, S. Lapin, M. Flury, Capillary Forces between Sediment Particles and an Air-Water Interface, Environ. Sci. Technol. 46 (2012) 4411-4418.

[27] A.A. Keller, S. Sirivithayapakorn, Transport of colloids in unsaturated porous media: Explaining large-scale behavior based on pore-scale mechanisms, Water Resour. Res. 40 (2004) W12403.

[28] C.J. van Oss, R.F. Giese, P.M. Costanzo, DLVO and Non-DLVO Interactions in Hectorite, Clay. Clay. Miner. 38 (1990) 151-159.

[29] D. Grasso, K. Subramaniam, M. Butkus, K. Strevett, J. Bergendahl, A review of non-DLVO interactions in environmental colloidal systems, Reviews in Environmental Science and Biotechnology 1 (2002) 17-38.

[30] J.M. Wan, T.K. Tokunaga, Film straining of colloids in unsaturated porous media: Conceptual model and experimental testing, Environ. Sci. Technol. 31 (1997) 24132420.

[31] R.A. Akbour, J. Douch, M. Hamdani, P. Schmitz, Transport of kaolinite colloids through quartz sand: Influence of humic acid, Ca2+, and trace metals, J. Colloid Interf. Sci. 253 (2002) 1-8. 


\section{Tables}

Table 1. Surface properties of the SWNTs and the sand used in this work.

\begin{tabular}{ccc}
\hline Materials & $\begin{array}{c}\text { Electrophoretic mobility } \\
\left(\times \mathbf{1 0}^{-8} \mathbf{m}^{\mathbf{2}} /(\mathbf{V s})\right)\end{array}$ & $\begin{array}{c}\text { Zeta-potential } \\
(\mathbf{m V})\end{array}$ \\
\hline O-SWNTs & -2.69 & -36.44 \\
S-SWNTs & -3.30 & -42.23 \\
H-SWNTs & -3.34 & -45.20 \\
Fine Sand & -1.53 & -19.59 \\
Medium Sand & -1.54 & -19.73 \\
Coarse Sand & -2.15 & -27.56 \\
\hline
\end{tabular}


Table 2. Summary of sand column experimental conditions and model parameters.

\begin{tabular}{ccccccc}
\hline & & \multicolumn{6}{c}{ Model parameters } \\
\cline { 3 - 7 } Treatment & Moisture & $\begin{array}{c}\boldsymbol{v} \\
(\mathbf{c m} / \mathbf{m i n})\end{array}$ & $\boldsymbol{P e}$ & $\boldsymbol{D a}$ & $\begin{array}{c}\boldsymbol{L}_{\text {max }} \\
(\mathbf{m})\end{array}$ & $\boldsymbol{R}^{2}$ \\
\hline Medium sand & & & & & & \\
O-SWNTs & & & & & & \\
Saturated & 0.39 & 1.8 & 33.1 & 0.025 & 46 & 1.00 \\
Unsaturated & 0.21 & 3.1 & 14.7 & 0.037 & 31 & 0.99 \\
Unsaturated & 0.13 & 1.3 & 27.7 & 0.133 & 9 & 0.99 \\
S-SWNTs & & & & & & \\
Saturated & 0.39 & 2.0 & 22.5 & 0.035 & 33 & 1.00 \\
Unsaturated & 0.21 & 3.7 & 24.1 & 0.051 & 23 & 1.00 \\
Unsaturated & 0.13 & 1.4 & 21.0 & 0.068 & 17 & 1.00 \\
H-SWNTs & & & & & & \\
Saturated & 0.39 & 1.8 & 43.2 & 0.028 & 41 & 1.00 \\
Unsaturated & 0.21 & 3.1 & 37.5 & 0.018 & 64 & 1.00 \\
Unsaturated & 0.13 & 1.2 & 36.9 & 0.026 & 44 & 1.00
\end{tabular}

Fine sand

O-SWNTs

$\begin{array}{lcccccc}\text { Saturated } & 0.38 & 2.1 & 22.6 & 0.108 & 11 & 1.00 \\ \text { Unsaturated } & 0.33 & 2.4 & 18.6 & 0.094 & 12 & 0.99 \\ \text { Unsaturated } & 0.29 & 0.6 & 24.5 & 0.157 & 7 & 0.99 \\ \text { S-SWNTs } & & & & & & \\ \quad \text { Saturated } & 0.38 & 0.5 & 100.8 & 0.073 & 16 & 1.00 \\ \text { Unsaturated } & 0.33 & 2.4 & 60.9 & 0.078 & 15 & 1.00 \\ \text { Unsaturated } & 0.29 & 0.7 & 77.2 & 0.097 & 12 & 1.00 \\ \text { H-SWNTs } & & & & & & \\ \text { Saturated } & 0.38 & 2.1 & 30.3 & 0.009 & 123 & 0.99 \\ \text { Unsaturated } & 0.33 & 2.5 & 33.6 & 0.016 & 74 & 0.99 \\ \text { Unsaturated } & 0.29 & 0.7 & 49.4 & 0.026 & 45 & 1.00\end{array}$

Coarse sand

O-SWNTs

$\begin{array}{lcccccc}\text { Saturated } & 0.41 & 1.6 & 37.8 & 0.018 & 63 & 1.00 \\ \text { Unsaturated } & 0.11 & 4.6 & 18.4 & 0.146 & 8 & 1.00 \\ \text { Unsaturated } & 0.09 & 1.1 & 18.1 & 0.250 & 5 & 1.00 \\ \text { S-SWNTs } & & & & & & \\ \text { Saturated } & 0.41 & 2.0 & 41.7 & 0.023 & 51 & 1.00 \\ \text { Unsaturated } & 0.11 & 5.7 & 12.1 & 0.064 & 18 & 1.00 \\ \text { Unsaturated } & 0.09 & 2.2 & 10.1 & 0.069 & 17 & 1.00 \\ \text { H-SWNTs } & & & & & & \\ \text { Saturated } & 0.41 & 1.7 & 48.4 & 0.008 & 139 & 0.99 \\ \text { Unsaturated } & 0.11 & 5.1 & 49.3 & 0.001 & 845 & 1.00 \\ \text { Unsaturated } & 0.09 & 1.4 & 25.5 & 0.040 & 29 & 1.00\end{array}$

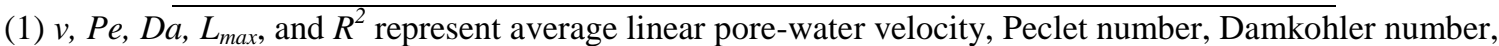
maximum travel distance, and coefficient of determination, respectively. 


\section{Figure Captions}

Figure 1. FTIR spectra of pristine SWNTs and O-SWNTs.

Figure 2. Stability of surface modified SWNTs in aqueous solutions.

Figure 3. Temporal changes of surface modified SWNT concentrations at the eight sampling ports within the bubble column.

Figure 4. Transport of surface modified SWNTs in medium sand columns under saturated (volumetric moisture content, 0.39) and unsaturated (volumetric moisture content, 0.21 and 0.13 ) conditions. Symbols are experimental data and lines are model results.

Figure 5. Transport of surface modified SWNTs in fine sand columns under saturated (volumetric moisture content, 0.38) and unsaturated (volumetric moisture content, 0.33 and 0.29) conditions. Symbols are experimental data and lines are model results.

Figure 6. Transport of surface modified SWNTs in coarse sand columns under saturated (volumetric moisture content, 0.41) and unsaturated (volumetric moisture content, 0.11 and 0.09) conditions. Symbols are experimental data and lines are model results. 
Figure 1

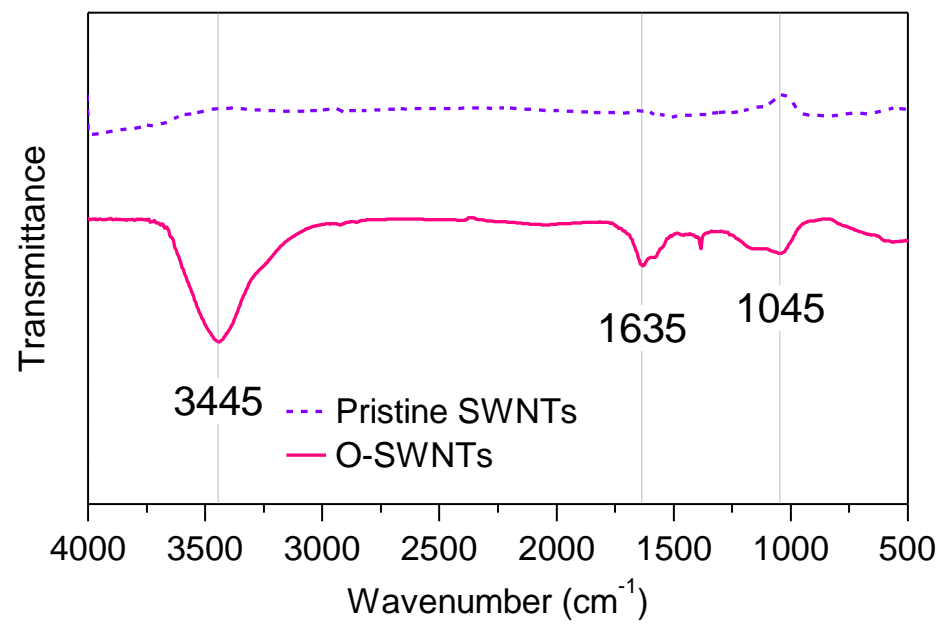


Figure 2

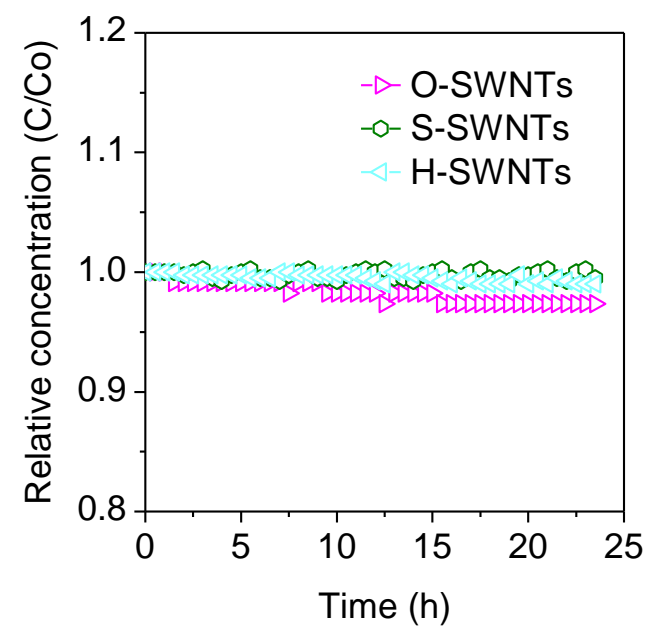




\section{Figure 3}

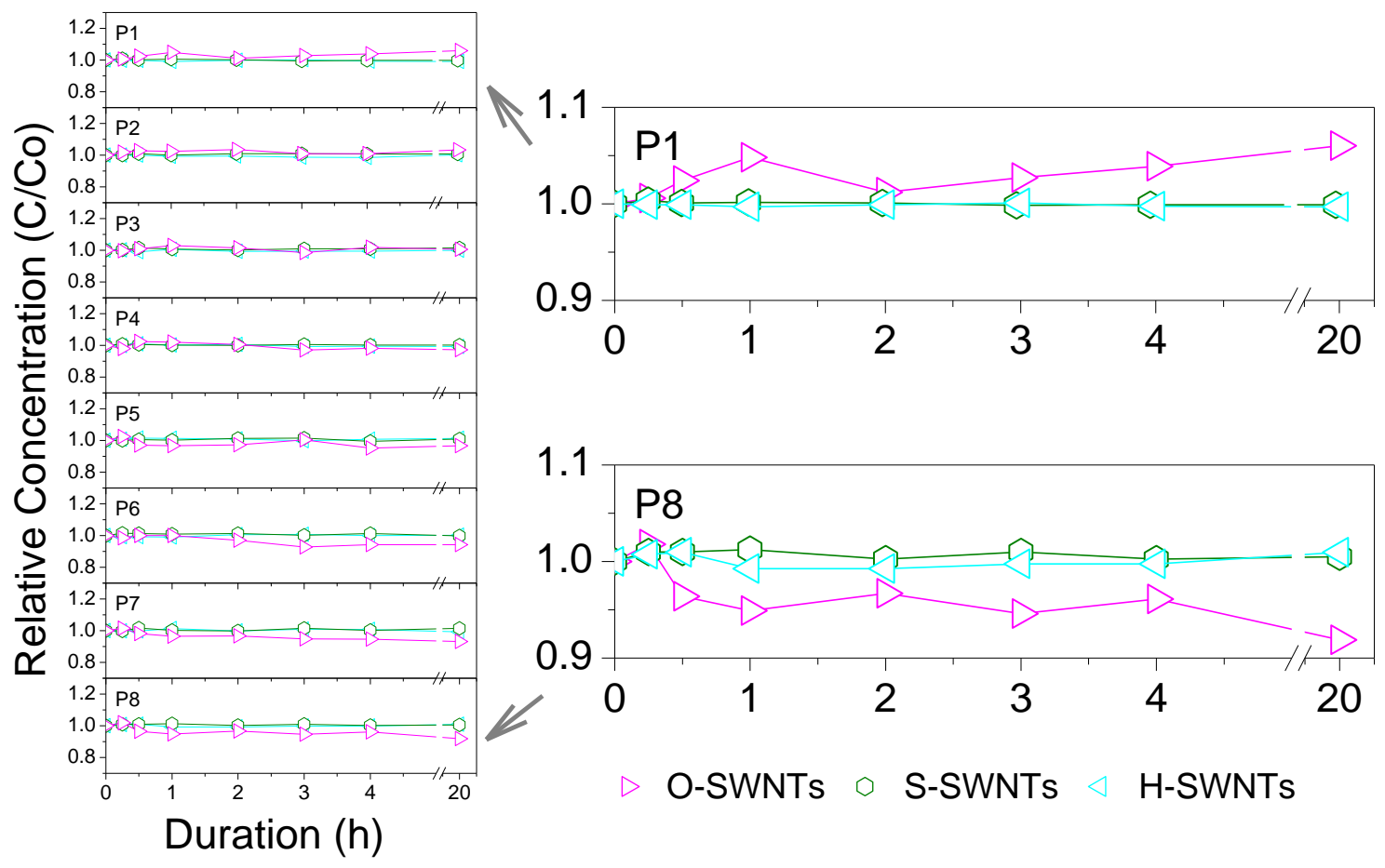




\section{Figure 4}
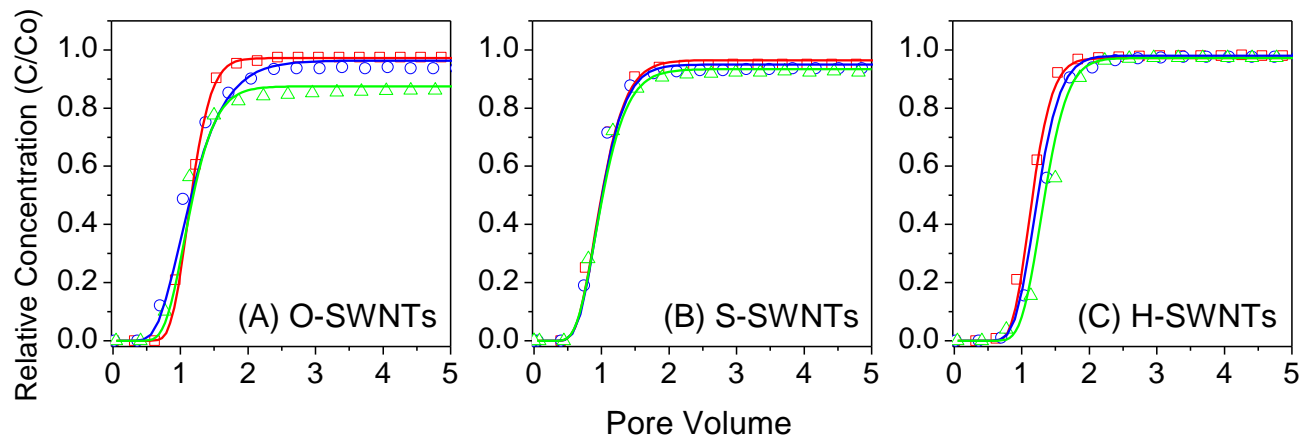

Medium sand: $\square$ Saturated (0.39) $\circ$ Unsaturated (0.21) $\triangle$ Unsaturated (0.13) 


\section{Figure 5}
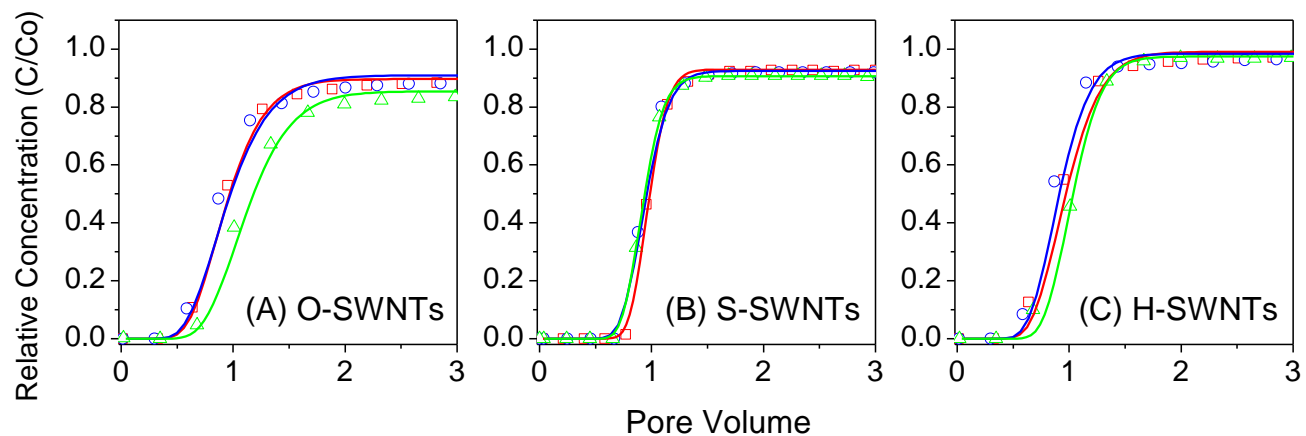

Fine sand: $\square$ Saturated (0.38) $\quad$ Unsaturated (0.33) $\Delta$ Unsaturated $(0.29)$ 


\section{Figure 6}
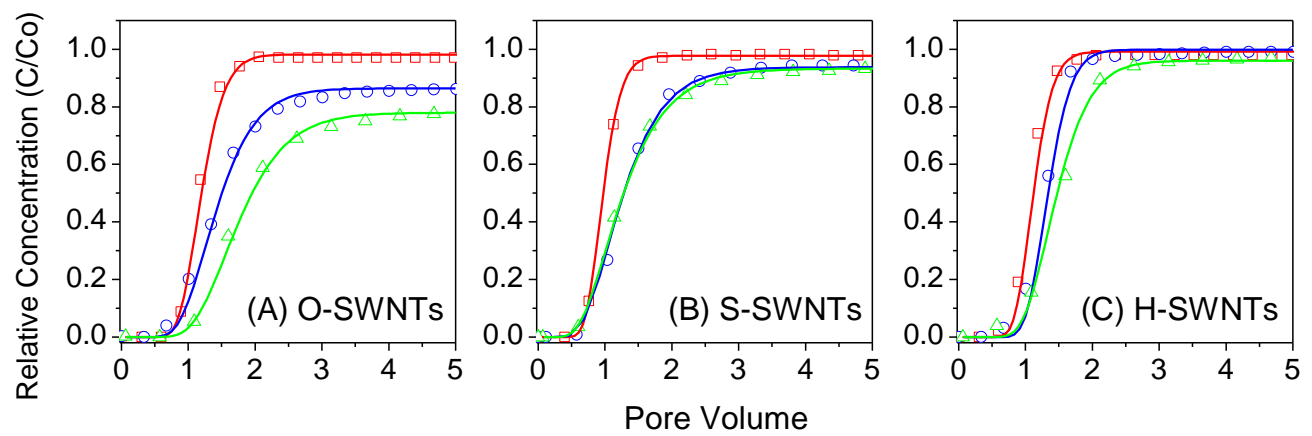

Coarse sand: $\square$ Saturated (0.41) $\triangle$ Unsaturated (0.11) $\triangle$ Unsaturated (0.09) 